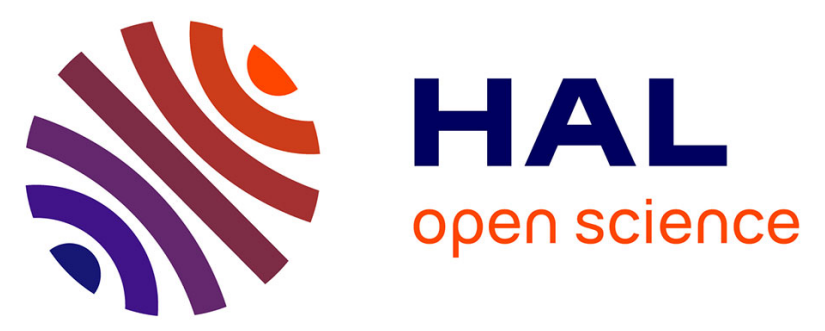

\title{
Design and Modeling of a Successive Approximation ADC for the Electrostatic Harvester of Vibration Energy
}

\author{
Raouf Khalil, Andrii Dudka, Dimitri Galayko, Ramy Iskander, Philippe
}

Basset

\section{- To cite this version:}

Raouf Khalil, Andrii Dudka, Dimitri Galayko, Ramy Iskander, Philippe Basset. Design and Modeling of a Successive Approximation ADC for the Electrostatic Harvester of Vibration Energy. BMAS 2010

- IEEE International Behavioral Modeling and Simulation Conference, Sep 2010, San Jose, United States. pp.57-62, 10.1109/BMAS.2010.6156599 . hal-00590893

\section{HAL Id: hal-00590893 \\ https://hal.science/hal-00590893}

Submitted on 5 May 2011

HAL is a multi-disciplinary open access archive for the deposit and dissemination of scientific research documents, whether they are published or not. The documents may come from teaching and research institutions in France or abroad, or from public or private research centers.
L'archive ouverte pluridisciplinaire HAL, est destinée au dépôt et à la diffusion de documents scientifiques de niveau recherche, publiés ou non, émanant des établissements d'enseignement et de recherche français ou étrangers, des laboratoires publics ou privés. 


\section{Design and Modeling of a Successive Approximation ADC for the Electrostatic Harvester of Vibration Energy}

\author{
Raouf Khalil, Andrii Dudka, \\ Dimitri Galayko, Ramy Iskander \\ Paris-VI university, \\ LIP6 laboratory, b.c. 167, \\ 4 , place Jussieu \\ 75252 Paris, \\ France \\ raouf.khalil-ayad@lip6.fr
}

\author{
Philippe Basset \\ Université Paris-Est, ESYCOM, \\ EA 2552, ESIEE \\ 2, Boulevard Pascal, BP 99 \\ 93162 Noisy le Grand, \\ France \\ p.basset@esiee.fr
}

\begin{abstract}
This paper presents a functional design and modeling of a successive approximation analog-to-digital converter (ADC) and its application in the conditioning circuit of the vibration energy harvester. The paper published on BMAS'09 highlighted the necessity of a smart digital control to calibrate the system, which requires in turn an ultra low power supplied ADC. The harvester and the ADC are designed using a CMOS $0.35 \mu \mathrm{m}$ High Voltage technology and modeled in a mixed VHDL-AMS/ELDO environment. The supply voltage of the ADC is $2.5 \mathrm{~V}$. The whole system was simulated using a mixed signal AdvanceMS simulator.
\end{abstract}

\section{Keywords}

Energy harvesting, successive approximation ADC, VHDLAMS, calibration, MEMS

\section{INTRODUCTION}

Modern self-powered autonomous electronic micro-systems, such as wireless micro-sensor network, embedded monitoring systems, biomedical implants, tend to incorporate more functions into smaller surfaces of devices and also are required to operate for a relatively long time without human intervention. On the other hand, the evolution in power source development is not following the same trend: the power supply sources still occupy an important part of size and weight of the system. The energy capacity, as well as lifetime of the existing sources is limited, and alternative energy sources are needed to overcome these bottlenecks. The autonomy of the devices can be extended by extracting power from ambient energy sources such as light, temperature gradients, mechanical vibration and others. Longlasting, self-renewable, chip-compatible energy harvesting sources are therefore a subject of research for a growing number of scientists and engineers. This study is focused on conversion of the energy of external mechanical vibrations into electrical energy with a use of an electrostatic transducer, which operates as a variable capacitor implemented in a MEMS technology. Harvesting the vibration energy with the capacitive transducer requires complex conditioning electronics for managing the transducer operation and for interfacing the transducer with the load. Harvester, operating under conditions of variable vibration parameters, claims a "smart" power management and adaptive control of the system operation. Our previously published work in
BMAS'09 [1] addressed the system adaptation to the variable amplitude of vibrations and, consequently, proposed an adaptive operation algorithm which was implemented in a power management control block and modeled in VHDLAMS.

The need of measurements of the voltages of the conditioning circuit used for the "smart" control electronics managing the operation of conditioning circuit and the interface with the load requires an analog-to-digital converter with ultra low power consumption. Power saving can be achieved in ADC through the selection of a suitable low-power ADC. The successive approximation ADC is known as one of the best candidates in terms of low-power [2].

This paper presents the design of a successive approximation ADC, and its modeling in the context of the conditioning circuit presented in [1]. The most critical blocks for the power consumption of the ADC are described at the electrical netlist level (the comparator and the digital-toanalog converter, DAC), and the control unit is described behaviourally in VHDL-AMS language. The main goal of the mixed modeling of the whole system (ADC+conditioning circuit) is an estimation of the power losses associated with the electromechanical power conversion and with the measures of the electrical state of the conditioning circuit needed for the calibration. This estimation doesn't take into account the consumption related with purely digital blocks.

In section 2 , we summarize the basic architecture and the operation of the harvester as well as the calibration technique allowing the adaptation of the system to the vibration parameters. In section 3 , we present the architecture of the successive approximation ADC and we explain the implementation and modeling approach for each block. In section 4, the connection of harvester system with the ADC, calibration technique is discussed and simulation results of the whole system are shown.

\section{HARVESTER OPERATION}

An electrostatic energy harvester consists of a mechanical resonator, an electromechanical transducer (based on MEMS technology) [3] and an electrical conditioning circuit managing the operation of the transducer and providing an interface with the load [1]. One of the possible architecture 
of conditioning circuit, initially proposed by Yen [4], is composed of two main parts: a charge pump and a flyback circuit controlled by a switch SW (Fig. 1). The charge pump circuit transfers electrical charges from a large initially pre-charged capacitor $C_{r e s}$ toward a small temporary storage $C_{\text {store }}$ with the use of variable transducer capacitor $C_{v a r}$. The energy for this charge pumping comes from the mechanical domain through the variations of the transducer capacitor. During the pumping, the converted energy is stored in the $C_{\text {store }}$ capacitor, the $C_{\text {store }}$ voltage $U_{\text {store }}$ increases and $C_{\text {res }}$ voltage $U_{\text {res }}$ decreases very slightly (since $C_{\text {res }}>>C_{\text {store }}$ ). It has been shown that the energy conversion is maximal for a particular range $\left(U_{1}, U_{2}\right)$ of $U_{\text {store }}[1]$, and when $U_{\text {store }}$ reaches the upper border of this range $U_{2}$, a part of the charges of $C_{\text {store }}$ must be returned to $C_{\text {res }}$, so to put $U_{\text {store }}$ at the low limit $U_{1}$ of this range. This is performed by the inductive flyback circuit. Topologically, the flyback circuit is similar to a Buck DC/DC converter, but with an output voltage $U_{r e s}$ needed to be pulled up as efficiently as possible using the energy of $C_{\text {store }}$. As soon as $U_{\text {store }}$ reaches $U_{2}$, the

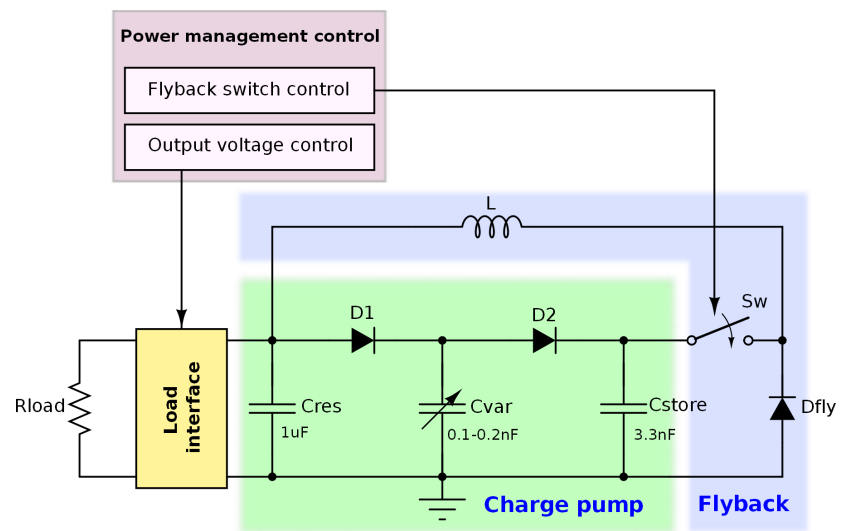

Figure 1: Conditioning circuit architecture of the vibration energy harvester

switch SW is set on by external control blocks and $C_{\text {store }}$ starts to discharge on $C_{r e s}$ through the inductor. When $U_{\text {store }}$ is reduced to $U_{1}$, the switch becomes of $f$ and the inductor discharges on $C_{r e s}$ through the diode D3. After this, a new charge pump operation cycle starts. From theoretical investigation [5] $U_{1}$ and $U_{2}$ parameters are calculated as:

$$
\begin{aligned}
& U_{1}=U_{\text {res }}+0.1\left(U_{\text {store } \max }-U_{\text {res }}\right), \\
& U_{2}=U_{\text {res }}+0.6\left(U_{\text {store max }}-U_{\text {res }}\right) .
\end{aligned}
$$

Here, $U_{\text {store } \max }$ is the saturation voltage of the charge pump given by [4]:

$$
U_{\text {store } \max }=\frac{C_{\max }}{C_{\min }} U_{\text {res }}
$$

where $C_{\max }$ and $C_{\min }$ are the maximal and minimal values which takes the transducer capacitance. From eq. (2), $U_{\text {store } \max }$ depends on the ratio between $C_{\max }$ and $C_{\min }$ that are, in turn, related to the resonator vibration parameters such as frequency and amplitude, which usually vary in the time. Consequently, $U_{\text {store } \max }$ voltage is not a fixed value and moreover can not be measured directly since during charge pumping $U_{\text {store }}$ never reaches the saturation [1].
In the paper presented on BMAS'09 we showed, however, that $U_{\text {store } \max }$ can be measured in an ad-hoc calibration cycle, which repeats periodically (much more rarely then a charge pump+flyback cycle).

During the calibration cycle, the flyback is deactivated and the charge pump runs freely up to the saturation. At the saturation, the $U_{\text {store }}$ value can be measured and used as $U_{\text {store } \max }$ in $U_{1}$ and $U_{2}$ calculation. The operation of the calibration cycle can be summarized as follow [1]: firstly, the charge pump is initialized ( $U_{\text {store }}$ resets to $\left.U_{\text {res }}\right)$, secondly, periodic measurements of $U_{\text {store }}$ during the charge pumping are provided. At each measurement, $U_{\text {store }}$ is compared with the previously measured value. As soon as the difference $\Delta U$ between two neighboring measured values of $U_{\text {store }}$ becomes lower than a predefined minimal value $\Delta U_{m i n}$, it is concluded that $U_{\text {store }}$ is saturated and the last measured value is considered as $U_{\text {store } \max }$. The calibration cycle ends by the updating of $U_{1}$ and $U_{2}$. Hence, between two calibration cycles, the system operates with optimized $U_{1}$ and $U_{2}$ switching parameters, which are likely to improve the energy yield of the harvester regarding to the variation of ambient conditions.

In order to calculate $U_{1}$ and $U_{2}$ during the system calibration, we need to measure $U_{\text {store max }}$ and $U_{\text {res }}$. Consequently, to interface analog voltages with the digital electric circuit we need to use an analog-to-digital converter.

Vibration energy harvesters provide low values of powers (tens microwatts), hence, the conditioning electronics, including ADC, should consume as few as possible. In this work, we demonstrate the design of the very low power consumption successive approximation ADC.

\section{SUCCESSIVE APPROXIMATION ADC ARCHITECTURE}

In Fig. 2, we can see how different parts of the successive approximation $\mathrm{ADC}$ are connected. In our topology, the signal is sampled in the first clock cycle and is converted in the next $N$ clock cycles, where $N$ is the number of bits. The sample and hold operation is achieved with the use of the DAC, when it is set in "sampling" mode. The DAC, allowing to achieve the successive approximation of the input voltage value, contains an array of binary weighted capacitors whose topology can be reconfigured by switches. The DAC output is connected to the negative input terminal of the comparator as it is shown in Fig. 3, while the common mode voltage $U_{c m}$ is connected to the positive input terminal and the comparator output is connected to the control unit. The control unit represents the successive approximation register (SAR) that controls the DAC switches allowing to switch capacitors terminals to the three reference voltages: the supply voltage $U_{d d}$, the common mode voltage $U_{c m}=U_{d d} / 2$ and the ground gnd. It generates the output digital word representing the digitally approximated input voltage. This architecture was described in details in [6].

\subsection{Successive approximation ADC stages}

This section describes stages of successive approximation ADC conversion, achieved in different modes. 


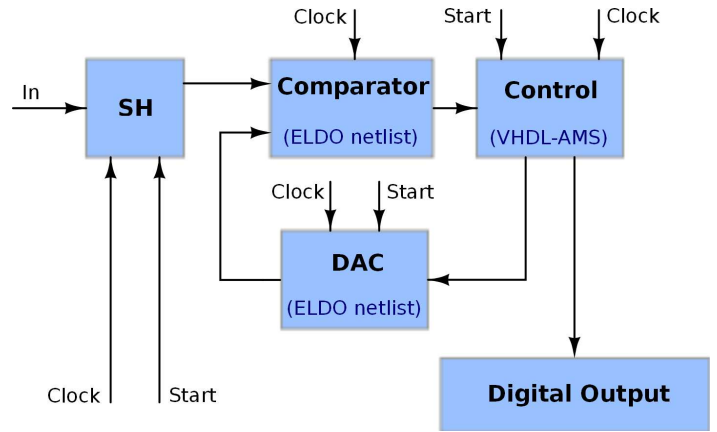

Figure 2: Successive approximation ADC architecture

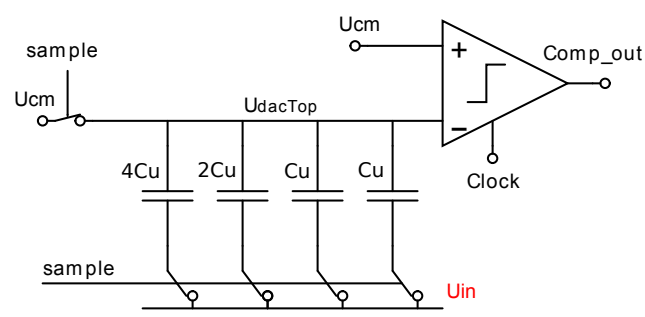

Figure 3: Sampling Mode in successive approximation ADC with 4 bits

In the first half cycle of the first clock cycle, the DAC capacitors bottom plates in the capacitor array are connected to the input signal $U_{i n}$, while the top plates are connected to $U_{d d} / 2$ : this is the "sampling" mode and it corresponds to the configuration shown in Fig. 3. In the second half of the clock cycle, all bottom plates are connected to $U_{d d} / 2$ : this is the inversion mode. After the first cycle, the control unit checks the comparator output (comp_out). If it is high, it connects the largest capacitor bottom plate to $U_{d d}$, if it is low, the control unit connects the largest capacitor bottom plate to gnd. This mode is called charge redistribution mode. The same is repeated with all capacitors in the array, in $N$ clock cycles. At $i^{\text {th }}$ clock cycle, the output of the comparator correspond to $i^{t h}$ bit of the output ADC word. This bit value is saved by the control unit and after the end of the charge redistribution mode, the digital output of the control unit is equal to the digitally approximated input voltage. In Fig. 4, we can see how the voltage of the DAC top plates changes in every mode on the example of 8-bit successive approximation ADC.

\subsection{Comparator Circuit}

The comparator in Fig. 5 is designed in CMOS $0.35 \mu \mathrm{m}$ technology. This is a semi-dynamic clocked architecture and it compares the output of the DAC $U_{D A C T o p}$ that is connected to the negative terminal and $U_{c m}$ that is connected to the positive terminal. Transistors M1 and M2 are used to amplify the input signal. Transistors M3, M4, M9 and M10 implement a couple of inverters connected so to be a flip-flop (back to back inverters). Transistors sizes are chosen with a low aspect ratio so as to reduce the power consumption and with a large area to reduce the offset mismatch. The comparator uses an internal clock equal to $2.5 \mathrm{KHz}$. Thus, using the resolution of the ADC $N=8$ bit, the sampling fre-
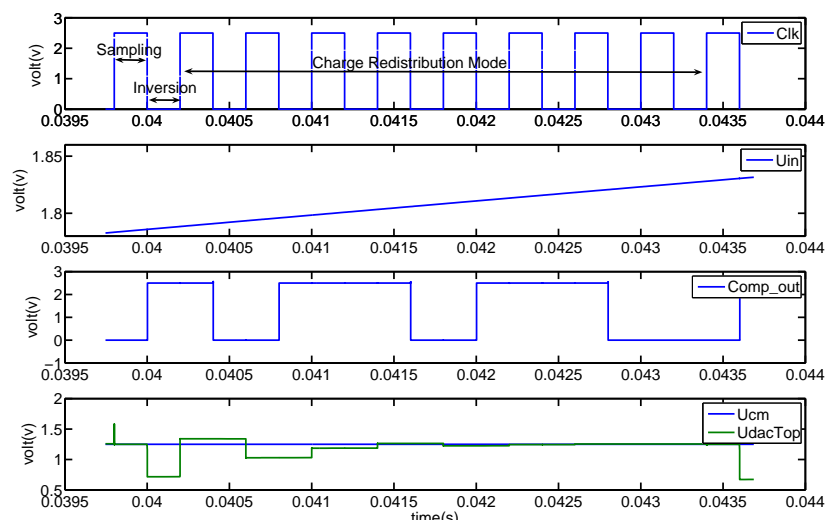

Figure 4: 8-bit successive approximation ADC modes

quency is $277.78 \mathrm{~Hz}(2.5 \mathrm{KHz} / \mathrm{N}+1)$, i.e the sampling time step equals to $3.6 \mathrm{~ms}$. The average current consumed in one conversion step equals to $0.5 \mu \mathrm{A}$. As shown in Fig. 6, the comparator resolution is lower than $U_{L S B}=9.8 \mathrm{mV}$. Both comparator outputs are connected to a latch to keep the comparator output stable for every clock cycle.

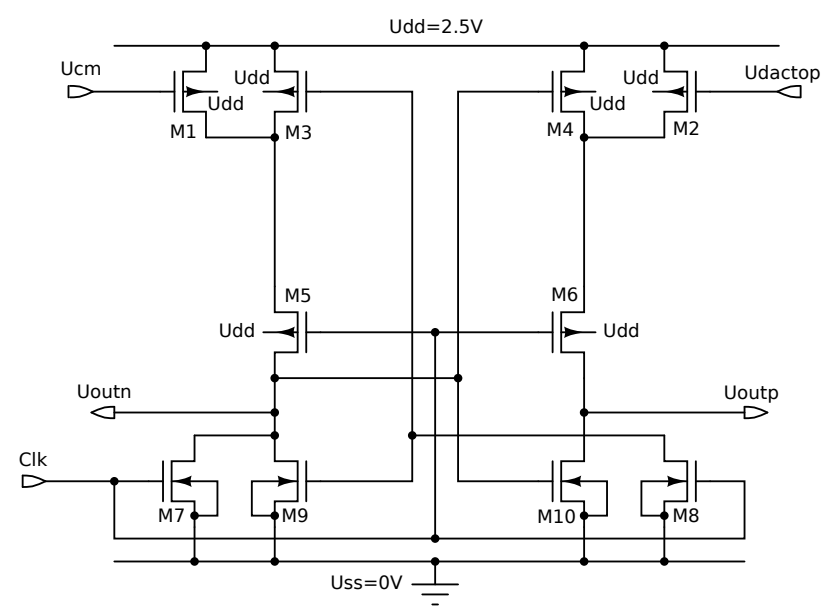

Figure 5: Comparator circuit
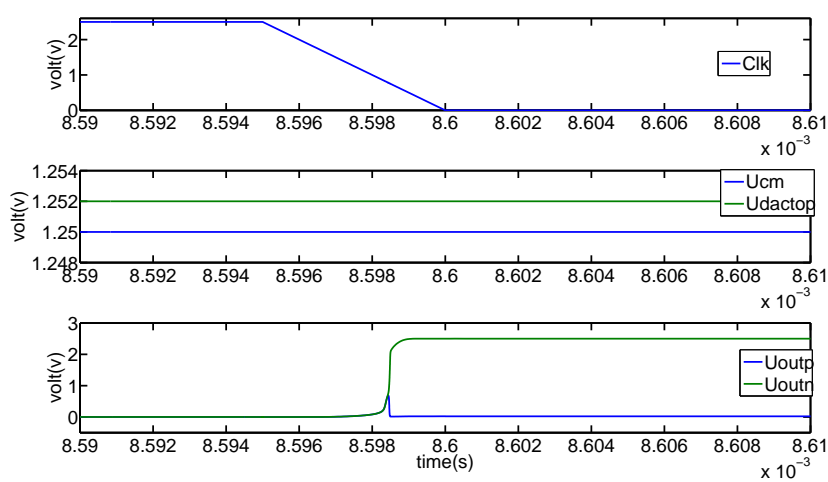

Figure 6: Comparator Resolution 


\subsection{Digital to Analog Converter Circuit}

The DAC is designed in CMOS $0.35 \mu \mathrm{m}$ technology and it contains 8 binary weighted capacitors in the array, the top plates are connected together. At each bottom plate, there are ideal switches to connect the bottom plate with the input signal and the three references as shown in Fig. 7. The choice of the unit capacitance value $\left(C_{u}\right)$ depends on the input capacitance of the comparator, the needed resolution and the sampling frequency.

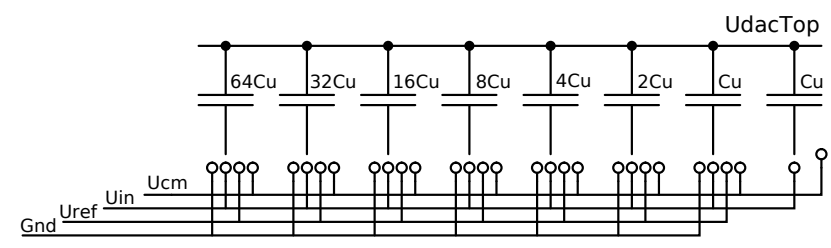

Figure 7: Differential DAC of a 8-bit successive approximation $\mathrm{ADC}$ with unit capacitance $C_{u}$

\subsection{Control Unit VHDL-AMS Model}

The output of the comparator is connected to the SAR control unit. At the sampling mode, the SAR control unit commands the switches so that the upper plates of the array are connected to $U_{d d} / 2$ and the bottom plates are connected to $U_{i n}$. Then, at inversion mode, it commands switches so that the bottom plates are connected to $U_{d d} / 2$. In each clock cycle of the charge redistribution mode, depending on the comparator output, the control unit decides either to connect the corresponding weighted capacitor bottom plate to $U_{d d}$ or to $g n d$. In this way, the control unit raises or reduces voltages on the top plates of the capacitors array by binary weighed level voltages $\left(U_{d d} / 4, U_{d d} / 8, \ldots, U_{d d} / 256\right)$, so achieving the successive approximation of the input voltage. This block was described in VHDL-AMS language as it is shown in the code below.

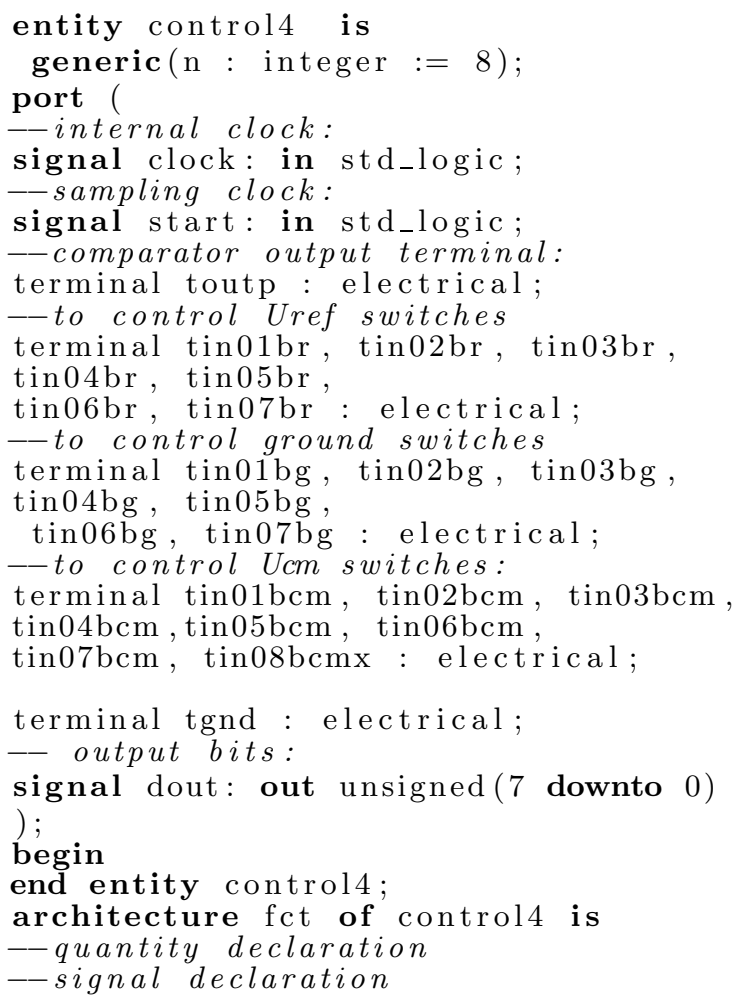

begin

p1: process

variable $\mathrm{i}$ : integer $:=\mathrm{n}$;

variable word : unsigned $((n-1)$ downto 0$)$;

begin

-_ disable all control signals

LOOP

wait on clock;

- first cycle in charge redistribution mode

if clock' event and clock='1' and start='0'

and $(\mathrm{i}=\mathrm{n})$ then

$\mathrm{i}:=\mathrm{n}-1$;

--connect the capacitors

--bottom plates to Ucm

in $02 \mathrm{bcm}<=2.5$

in $03 \mathrm{bcm}<=2.5$

in $04 \mathrm{bcm}<=2.5$;

in $05 \mathrm{bcm}<=2.5$

in $06 \mathrm{bcm}<=2.5$

in $07 \mathrm{bcm}<=2.5$;

in 08 bcmx $<=2.5$;

if (comp_out $>1$ ) then

word $(\mathrm{i}):={ }^{\prime}, 1, ;--m s b=1$

in $01 \mathrm{br}<=2.5 ;--$ connect the msb cap to Uref

else

$\operatorname{word}(\mathrm{i}):=0^{\prime} ;--m s b=0$

in $01 \mathrm{bg}<=2.5 ;-$ connect the msb cap to gnd end if ;

else

- second cycle in charge distribution mode if clock' event and clock =' 1 ' and start $=$ ' 0 ' and $(\mathrm{i}=\mathrm{n}-1)$ then

- connect the capacitors bottom plates to Ucm $\mathrm{i}:=\mathrm{i}-1$;

in $02 \mathrm{bcm}<=0.0$;

in $03 \mathrm{bcm}<=2.5$

in $04 \mathrm{bcm}<=2.5$;

in $05 \mathrm{bcm}<=2.5$

in $06 \mathrm{bcm}<=2.5$;

in $07 \mathrm{bcm}<=2.5$;

in 08 bcm $<=2.5$;

if (comp_out $>1$ ) then

word $(\mathrm{i}):={ }^{\prime} 1{ }^{\prime} ;-$ - 2nd bit $=1$

in $02 \mathrm{br}<=2.5 ;-$ connect the 2nd cap to Uref

else

word (i):= '0';-- 2nd bit $=0$

in $02 \mathrm{bg}<=2.5 ;-$ connect the 2 nd cap to gnd end if ;

else

- this algorithm is repeated

- to $i=n-r$

- last cycle in charge distribution mode

if clock'event and clock =' 1 ' and start'event and start $=$ ' 1 ' and $(\mathrm{i}=\mathrm{n}-7)$ then

--connect the capacitors

-bottom plates to Ucm

$\mathrm{i}:=\mathrm{i}-1$;

in 08 bcmx $<=2.5$;

if (comp_out $>1$ ) then

word (i ): $={ }^{\prime} 1, ;--l s b$ bit $=1$

else

word $(\mathrm{i}):={ }^{\prime} 0^{\prime} ;--l s b$ bit $=0$

end if ;

-- we get the new output bits

-at the end of the conversion

dout $<=$ word

$\mathrm{i}:=\mathrm{n}$;

--disable all control signals

end loop ;

end process ;

end architecture fct; 


\section{APPLICATION OF SAR ADC INTO THE HARVESTER CONDITIONING CIRCUIT}

In this section, we present the use of the designed successive approximation ADC in the conditioning circuit of harvester, whose model architecture is shown in Fig. 8. The ADC measures only the $U_{\text {store }}$ voltage, whereas the value of $U_{\text {res }}$ can be found from the determined value of the $U_{\text {store }}$ converted voltage as we will explain later in this section. For optimal operation, the harvester requires the high voltage on $C_{\text {store }}$ [1], hence, to interface the low-voltage ADC input with the high-voltage $U_{\text {store }}$ node of the conditioning circuit, a voltage divider is needed. The output bits of the ADC are connected to the flyback switch control input which uses the measured values of $U_{\text {res }}$ and $U_{\text {store max }}$ to calculate $U_{1}$ and $U_{2}$. As soon as $U_{1}$ and $U_{2}$ are calculated, the switching events for the switch $\mathrm{Sw}$ are generated with the signals ${ } n_{2}$ or of $f_{2}$, as shown in Fig. 8. The flyback switch control activates the ADC with the signals on 1 or of $f_{1}$, so that the ADC operates only during the calibration cycle.

As it is shown in Fig. 8, the whole harvester system model is mixed: it contains VHDL-AMS models of the flyback switch control, the switch, the variable transducer capacitor with the resonator, the VHDL-AMS/ELDO model of the ADC, ELDO models of the voltage divider and of the rest analog components such as capacitors, inductor and diodes. In this

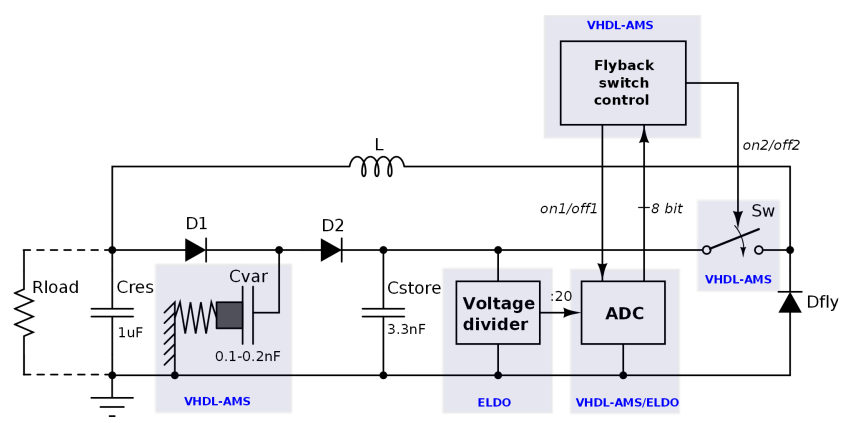

Figure 8: Functional schema of harvester system

section, firstly, we discuss the divider architecture and its operation, then the use of ADC during the calibration cycle and, at last, the whole system simulation results.

\subsection{Voltage Divider Circuit}

In present work the division factor of divider is 20 , since the maximal value for $U_{\text {store }}$ is limited by $50 \mathrm{~V}$ and the voltage supplied by the ADC is $2.5 \mathrm{~V}$. The proposed divider consists of 2 resistors connecting in series, 2 switches and an output capacitor as shown in Fig. 9. At every sample, resistors divide $U_{\text {store }}$ by 20 , and the divided voltage is stored in the capacitor $C_{d i v}$, so that the successive approximation ADC can sample this value. The clock used in the divider (CLK_ DIV) has the same sampling frequency but with the smaller pulse width to decrease the power consumption.

The relation between $U_{\text {store }}$ and the divider output $U_{\text {out_div }}$ can be calculated based on equation:

$$
\frac{U_{\text {store }}}{U_{\text {out_div }}}=\frac{R_{1}+R_{2}}{R_{2}}=20
$$

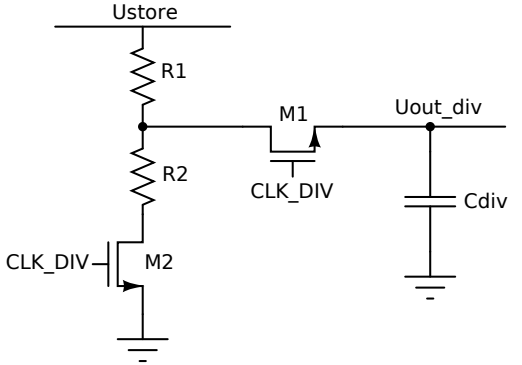

Figure 9: Voltage divider circuit interfacing the harvester with the $A D C$

Hence, from (3) we have $R_{1}=19 R_{2}$. The time of charging $C_{\text {div }}$ is determined by:

$$
\tau=R_{1} \| R_{2} \cdot C_{\mathrm{div}},
$$

The presented voltage divider circuit is designed in CMOS $0.35 \mu \mathrm{m}$ high voltage technology of Austrian Microsystem (AMS035HV), and modeled using the ELDO simulator.

\subsection{Calibration Using Successive Approximation ADC}

The calibration cycle starts with putting $U_{1}$ to a very low value (zero), so that $C_{\text {store }}$ voltage becomes equal to $C_{r e s}$ voltage. During $20 \mathrm{~ms} U_{\text {store }}$ remains at the $U_{\text {res }}$ value, which gives to the ADC enough time to measure it. After that, the flyback switch control orders the charge pumping to start, and $U_{\text {store }}$ starts to increase, up to the saturation. During this charge pumping, the voltage $U_{\text {store }}$ is measured with $3.6 \mathrm{~ms}$ sampling step. The goal of this measurement is to detect the $U_{\text {store } \max }$ value. This is done as described in the section 2 ; the digital values corresponding to the neighboring samples of $U_{\text {store }}$ are compared, and when there are 2 consecutive steps have the same value, their value is considered as measured $U_{\text {store max }}$ (it means that $\Delta U_{\text {min }}$ mentioned in section 2 equals to the ADC resolution). At the end of the calibration cycle the flyback switch control block turns off the ADC and $U_{1}$ as well as $U_{2}$ are calculated. Then, the normal mode (periodic charge pump+flyback cycles) starts till the next calibration cycle as it is shown in the Fig. 10.

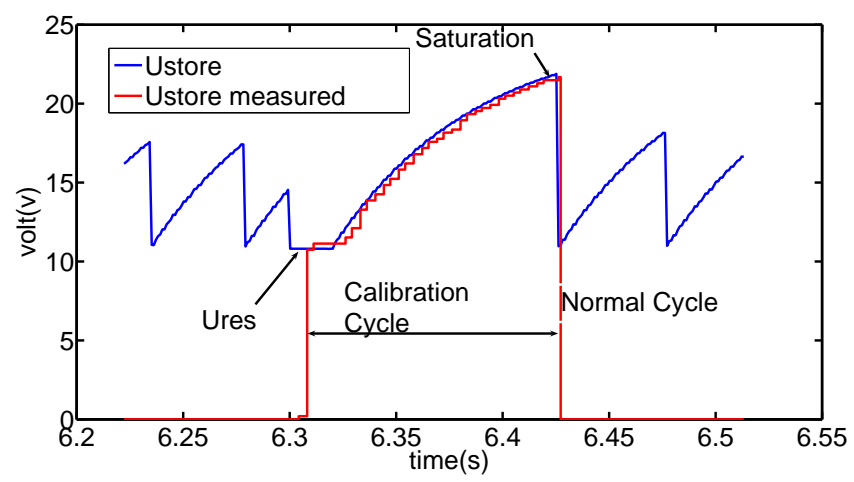

Figure 10: Calibration cycle of the harvester 


\subsection{Modeling Results}

In order to validate our study we created and simulated models of the divider and of the successive approximation $\mathrm{ADC}$ and connected them to the conditioning circuit model as it is shown in the Fig. 8. In this paper, we present simulation results which demonstrate the harvester operation with ADC during almost 11 seconds. Here, calibration cycles repeat every $900 \mathrm{~ms}$, such a low interval is chosen in order to reduce the modeling time, in reality the calibration phase should be less frequent (tens of seconds) to reduce the power consumed.

In the top part of the Fig. 11, $U_{\text {store }}$ is plotted together with the measured $U_{\text {store }}$ voltage. We can see that during the calibration cycle there is a good matching between them because of the good resolution of the ADC. In the bottom part of the Fig. 11, the real $U_{r e s}$ is plotted with the measured $U_{\text {res }}$ voltage. The increase in the real $U_{\text {res }}$ value is explained by the accumulation of the energy of the system (i.e., a normal harvester operation). As we see, $U_{\text {res }}$ is measured only once during the calibration cycle. In the normal operation mode between calibration cycles ADC is deactivated by the signal of $f_{1}$. The measured $U_{\text {store } \max }$ value is reset to 0 and the equivalent $U_{\text {res }}$ is saved until the next calibration cycle.
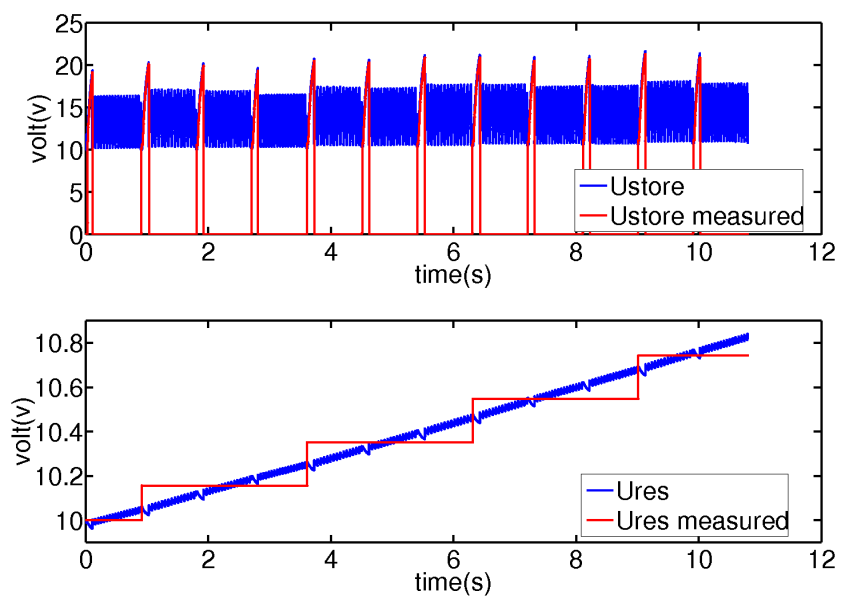

Figure 11: Simulation results of harvester operation using the SAR ADC model

\section{CONCLUSIONS}

In this paper, we presented the architecture design and modeling of the electrostatic vibration energy harvester with a successive approximation ADC. The use of such ADC in energy harvester application is suitable for a low power consumption. The whole system of the harvester including the SAR ADC is simulated with VHDL-AMS/ELDO mixed model using the cadence environment. Simulation results of the harvester with the use of 8-bit SAR ADC demonstrate a good matching between $U_{\text {store }}$ and the measured from ADC $U_{\text {store_mes }}$ during the calibration cycle. The estimated power consumption of the ADC is around $1.25 \mu \mathrm{W}$ for one step conversion. The comparator is the dominating block in power consumption of ADC with the used sampling frequency and 8-bit resolution, whereas the DAC has much less contribution. The modeling approach consisting in mixing the description levels and the disciplines is very efficient; the VHDL-AMS/ELDO mixed modeling appears as the best solution for low-level design of electrical interfaces for energy conversion.

\section{ACKNOWLEDGEMENTS}

This work is funded by the French National Research Agency (ANR).

\section{REFERENCES}

[1] Andrii Dudka, Dimitri Galayko, and Philippe Basset. Vhdl-ams modeling of adaptive electrostatic harvester of vibration energy with dual-output dc-dc converter. In IEEE International Behavioral Modeling and Simulation Conference (BMAS 2009).

[2] Michael D. Scott, Bernhard E. Boser, and Kristofer S. J. Pister. An ultra low-energy adc for smart dust. IEEE JOURNAL OF SOLID-STATE CIRCUITS, JULY 2003.

[3] P. Basset, D. Galayko, A. M. Paracha, F. Marty, A. Dudka, and T. Bourouina. A batch-fabricated and electret-free silicon electrostatic vibration energy harvester. Journal of Micromechanics and Microengineering, 2009.

[4] B. C. Yen and J. H. Lang. A variable-capacitance vibration-to-electric energy harvester. IEEE TCAS, 53(2):288-295, february 2006.

[5] D. Galayko and P. Basset. Mechanical/electrical power-aware impedance matching for design of capacitive vibration energy harvester. In PowerMEMS2008 workshop, Sendai, 2008.

[6] Mootaz Allam. Systematic design of a successive approximation analog-to-digital converter. Master's thesis, Faculty of Engineering,Cairo University, Giza, Egypt, 2008. 